\title{
The impact of the COVID-19 pandemic on negative symptoms in individuals at clinical high-risk for psychosis and outpatients with chronic schizophrenia
}

\author{
Gregory P. Strauss ${ }^{1} \cdot$ Kelsey I. Macdonald ${ }^{1} \cdot$ Ivan Ruiz $^{1} \cdot \operatorname{lan}$ M. Raugh ${ }^{1} \cdot$ Lisa A. Bartolomeo ${ }^{1} \cdot$ Sydney H. James ${ }^{1}$
}

Received: 21 January 2021 / Accepted: 30 March 2021 / Published online: 21 April 2021

(c) Springer-Verlag GmbH Germany, part of Springer Nature 2021

\begin{abstract}
Negative symptoms are core features of schizophrenia-spectrum disorders that are frequently observed across all phases of illness. By their nature, COVID-19 social isolation, physical distancing, and health precautions induce behavioural aspects of negative symptoms. However, it is unclear whether these prevention measures also lead to increases in experiential negative symptoms, whether such effects are equivalent across individual negative symptom domains, and if exacerbations occur equivalently across phases of illness. The current study compared negative symptom severity scores obtained during the pandemic to pre-pandemic assessments in two samples: (1) outpatients with chronic schizophrenia (SZ: $n=32)$ and matched healthy controls (CN: $n=31)$ and (2) individuals at clinical high risk for psychosis (CHR: $n=25)$ and matched CN $(n=30)$. Pre-pandemic ratings of negative symptoms were clinically elevated in SZ and CHR groups, which did not differ from each other in severity. In SZ, ratings obtained during the pandemic were significantly higher than pre-pandemic ratings for all 5 domains (alogia, blunted affect, anhedonia, avolition, and asociality) and item-level analyses indicated that exacerbations occurred on both experiential and behavioral symptoms of anhedonia, avolition, and asociality. In contrast, CHR only exhibited increases in anhedonia and avolition items during the pandemic compared to pre-ratings. Findings suggest that negative symptoms should be a critical treatment target during and after the pandemic in the schizophrenia spectrum given that they are worsening and critically related to risk for conversion, functional outcome, and recovery.
\end{abstract}

Keywords Attenuated psychosis syndrome $\cdot$ Prodrome $\cdot$ Coronavirus $\cdot$ Pandemic

\section{Introduction}

COVID-19, an infectious disease caused by acute respiratory syndrome coronavirus 2 , purportedly originated in Wuhan, China in December, 2019. By March, 2020 it had emerged as a global pandemic. As of December, 2020, COVID-19 has resulted in over 73 million infections and 1.6 million deaths worldwide (google.com 12/15/20). Although the COVID19 pandemic has had an unprecedented toll on human life and the economy, it is predicted to also have a profound long-term effect on global mental health [15, 27, 31, 46, 56]. Preliminary evidence has supported these expectations, indicating increased rates of PTSD, depression, and anxiety

Gregory P. Strauss

gstrauss@uga.edu

1 Department of Psychology, University of Georgia, 125 Baldwin St., Athens, GA 30602, USA in the frontline healthcare workers and COVID-19 survivors $[5,13,37,41,55,67,72]$.

Effects of COVID-19 on those with pre-existing mental illnesses have yet to receive substantial empirical attention. However, a mental health crisis has been predicted in the aftermath of the pandemic $[15,27,56]$. This may be particularly true of psychotic disorders, which are generally considered the most serious form of mental illness and associated with high disability weights [9]. Clinical case reports and commentaries have posited that the COVID-19 pandemic will lead to widespread reductions in service utilization and subsequent increases in acute symptom exacerbations in those who had already been diagnosed with a psychotic disorder prior to the pandemic [35, 40, 44]. There is also fear that reduced access to healthcare, social isolation, and reduced physical activity may lead those at clinical high risk (CHR) for developing psychosis to transition to illness onset $[6,14,17]$. Although clinical reports are alarming, empirical evidence for the detrimental effects of the COVID-19 
pandemic on schizophrenia-spectrum symptoms in SZ and CHR populations has yet to be demonstrated relative to prepandemic data. Such investigations are warranted given that data collected during the pandemic indicates higher rates of attenuated psychosis in the general population compared to what would be expected [15, 39, 71].

The current study provides an initial report of findings from the University of Georgia PACE Study (Psychosis Assessment of COVID-19 Effects), an online investigation designed to evaluate COVID-19-related changes in symptom severity and their moderators in those with chronic schizophrenia (SZ) and individuals at clinical high risk for psychosis (CHR). This manuscript focuses on the effects of the COVID-19 pandemic on negative symptoms specifically (i.e., reductions in normative emotion, motivation, and behavior). Negative symptoms are core features of schizophrenia-spectrum disorders that are frequently observed across all phases of illness $[8,59]$. The effect of the COVID19 pandemic on negative symptoms is presently unclear. Therefore, it is vital to determine whether these symptoms have been exacerbated compared to pre-pandemic times, because they are so critically linked to poor communitybased functioning, reduced rates of recovery, low psychological well-being, and illness liability [19, 49, 60, 61,62, 64].

To flatten the curve, reduce burden on the healthcare system, and decrease the number of new infections and deaths, societies throughout the world have implemented shelter-inplace orders, quarantine, self-isolation, and physical/social distancing policies [18, 30, 36, 38, 45, 50, 54, 68]. By their very nature, these prevention measures induce behavioral aspects of negative symptoms. For example, they decrease the frequency of in-person social interactions (asociality), hinder the pursuit of pleasurable recreational activities (anhedonia), and limit engagement in typical goal-directed activities (avolition). Given that cognitive behavior therapy interventions are thought to achieve their (modest) beneficial effects on experiential components of negative symptoms by first enhancing behavioral activation [10, 23, 24, 25, 26, $34,52]$, it stands to reason that a widespread manipulation such as the pandemic that severely limits behavioral initiation might induce or exacerbate experiential negative symptoms. However, it is unclear whether such pandemic-driven effects might be expected to only occur in those with the most severe negative symptom profiles (i.e., chronic SZ), those with attenuated symptoms (i.e., CHR), or the population more generally (i.e., $\mathrm{CN}$ ).

In the current study, clinical ratings on the Brief Negative Symptom Scale (BNSS: [32] collected during the pandemic were compared with pre-pandemic ratings in outpatients with chronic schizophrenia or schizoaffective disorder (SZ), individuals at clinical high risk for psychosis (CHR) (i.e., those meeting criteria for a prodromal syndrome), and matched healthy controls $(\mathrm{CN})$. The following hypotheses were evaluated: (1) in pre-pandemic clinical ratings, SZ would have greater severity than CHR on all 5 BNSS domains, (2) both SZ and CHR groups would evidence increases in clinically rated negative symptoms during the pandemic compared to their pre-pandemic ratings, however, the magnitude of symptom exacerbation was predicted to be greater in SZ than CHR, reflecting illness stage effects, and (3) in both SZ and CHR, item level analyses would reveal that COVID-19-related symptom exacerbations occurred for both behavioral (i.e., frequency of social activity, motivated behavior, and pleasurable activity) and experiential (i.e., extent to which social interaction is desired, how motivated they are to engage in goal-directed activity, intensity of pleasure experienced) aspects of negative symptoms, suggesting that pandemic effects were not simply tautological environmentally induced behavioral reductions due to sheltering in place; 4) for ratings made during the pandemic, it was predicted that $\mathrm{SZ}$ and CHR would evidence higher scores than $\mathrm{CN}$ on all 5 negative symptom domains.

\section{Method}

\section{Participants}

Data were collected from two samples. Sample 1 consisted of outpatients with SZ and matched community $\mathrm{CN}$, whereas sample 2 consisted of CHR and matched community CN.

\section{Sample 1}

Participants included 32 outpatients meeting DSM5 criteria for schizophrenia or schizoaffective disorder (SZ) and 31 healthy controls $(\mathrm{CN})$. Participants with SZ were originally recruited for studies investigating mechanisms of negative symptoms that occurred prior to the COVID-19 pandemic $[3,11,12,51]$. Original recruitment occurred at outpatient mental health clinics in northeast Georgia, USA and online or printed advertisements. Patients were evaluated during periods of clinical stability as indicated by no self-reported change in medication type of dose within the past 4 weeks. Diagnosis was established via the SCID-5 [20]. SZ were generally in the chronic phase of illness, had experienced multiple episodes, and were experiencing mild-to-moderate symptoms.

Healthy control participants $(\mathrm{CN})$ were recruited through printed and online advertisements. $\mathrm{CN}$ completed a diagnostic interview, including the SCID-5 [20] and SCID-5-PD [21], and did not meet criteria for any current psychiatric disorder or schizophrenia-spectrum personality disorder. CN also had no family history of psychosis and did not meet lifetime criteria for psychotic disorders. 
No participants met criteria for substance use disorders (other than tobacco) and all denied lifetime history of neurological disorders associated with cognitive impairment (e.g., traumatic brain injury, epilepsy).

Individuals with $\mathrm{SZ}$ and $\mathrm{CN}$ did not significantly differ in age, parental education, sex, or ethnicity; however, SZ had lower personal education than $\mathrm{CN}$ (see Table 1).

\section{Sample 2}

Participants included 25 CHR participants and 30 healthy controls $(\mathrm{CN})$ who were originally recruited for studies examining reward processing mechanisms underlying negative symptoms and psychosis risk $[3,4,11]$. CHR participants were recruited from the Georgia Psychiatric Risk Evaluation Program (G-PREP), which receives referrals from local clinicians to perform diagnostic assessment and monitoring evaluations for youth displaying psychotic experiences. CHR participants were also recruited via online and printed advertisements. CHR participants were included if they met criteria for a prodromal syndrome on the Structured
Interview for Prodromal Syndromes [42]. All CHR participants met SIPS criteria for Attenuated Positive Symptoms (i.e., SIPS score of at least 3-5 on at least one positive symptom item, with a frequency of occurring at least once per week, 13 progression, 11 persistence, 1 partial remission. CHR participants did not meet lifetime criteria for a DSM5 psychotic disorder as determined via the SCID and two participants in the CHR sample had been prescribed an antipsychotic. No CHR met current criteria for a substance use disorder.

$\mathrm{CN}$ recruitment and inclusion/exclusion were identical to sample 1. CHR did not significantly differ from their matched $\mathrm{CN}$ group on age, sex, race, or parental education; however, CHR had lower personal education than $\mathrm{CN}$.

\section{Procedures}

During studies where initial recruitment occurred, SZ, CHR, and $\mathrm{CN}$ participants had all consented to be recontacted for future studies. Recontact was done via email, text message, or phone call to determine interest in participating in an

Table 1 Demographic Characteristics for Study 1 and Study 2

\begin{tabular}{|c|c|c|c|c|}
\hline Study 1 & $\begin{array}{l}\mathrm{SZ}(n=32) \\
\mathrm{M}(\mathrm{SD})\end{array}$ & $\begin{array}{l}\mathrm{CN}(n=31) \\
\mathrm{M}(\mathrm{SD})\end{array}$ & Test statistic $(\mathrm{Chi} \mathrm{Sq} / F)$ & $p$ value \\
\hline Age & $40.13(13.25)$ & $41.32(9.43)$ & 0.17 & 0.68 \\
\hline Personal education & $14.84(2.26)$ & $16.52(2.59)$ & 7.46 & 0.01 \\
\hline Parental Education & $14.86(2.67)$ & $14.78(2.75)$ & 0.01 & 0.92 \\
\hline$\%$ Female & $75 \%$ & $83.9 \%$ & 0.76 & 0.38 \\
\hline Race & & & 2.94 & 0.57 \\
\hline$\%$ White & $65.6 \%$ & $54.8 \%$ & & \\
\hline$\%$ Black & $15.6 \%$ & $19.4 \%$ & & \\
\hline$\%$ Hispanic & $12.5 \%$ & $16.1 \%$ & & \\
\hline$\%$ Asian & $0 \%$ & $6.5 \%$ & & \\
\hline$\%$ Other & $6.3 \%$ & $3.2 \%$ & & \\
\hline Days after shelter in place order & $129.06(22.97)$ & $122.3(13.73)$ & 1.98 & 0.16 \\
\hline Reported days sheltering in place & $145.17(31.97)$ & $136.9(16.48)$ & 1.54 & 0.22 \\
\hline Study 2 & $\begin{array}{l}\mathrm{CHR}(n=25) \\
\mathrm{M}(\mathrm{SD})\end{array}$ & $\begin{array}{l}\mathrm{CN}(n=30) \\
\mathrm{M}(\mathrm{SD})\end{array}$ & Test statistic $(\mathrm{Chi} \mathrm{Sq} / F)$ & $p$ value \\
\hline Age & $22.6(3.42)$ & $23.0(2.44)$ & 0.26 & 0.62 \\
\hline Personal education & $14.92(1.61)$ & $15.83(1.62)$ & 4.37 & 0.04 \\
\hline Parental Education & $15.88(2.01)$ & $15.62(1.89)$ & 0.22 & 0.64 \\
\hline$\%$ Female & $84 \%$ & $73.3 \%$ & 0.91 & 0.34 \\
\hline Race & & & 2.97 & 0.56 \\
\hline$\%$ White & $72 \%$ & $73.3 \%$ & & \\
\hline$\%$ Black & $8 \%$ & $6.7 \%$ & & \\
\hline$\%$ Hispanic & $8 \%$ & $6.7 \%$ & & \\
\hline$\%$ Asian & $8 \%$ & $13.3 \%$ & & \\
\hline$\%$ Other & $0 \%$ & $4 \%$ & & \\
\hline Days after shelter in place order & $124.16(19.69)$ & $120.67(15.26)$ & 0.55 & 0.46 \\
\hline Reported days sheltering in place & $139.43(22.86)$ & $141.27(17.58)$ & 0.11 & 0.74 \\
\hline
\end{tabular}


online study. Of those recontacted, 58.2\% of SZ, $62.5 \%$ of $\mathrm{CHR}$, and $70.1 \%$ of $\mathrm{CN}$ who were invited consented to complete the study (i.e., for total n's of $\mathrm{SZ}=32, \mathrm{CN}=31$; $\mathrm{CHR}=25, \mathrm{CN}=30$ ).

Participation occurred between July 9, 2020 and October 5, 2020. For historical context, the state of Georgia ordered shelter-in-place on April 3, 2020 to combat the COVID19 pandemic. The shelter-in-place order was lifted on April 30, 2020. At the time of study completion, the COVID-19 pandemic state of emergency was still in effect. COVID precautions (e.g., restrictions on certain businesses being open, mask wearing etc.) were widely in place throughout the period during which the online study was completed.

All participants completed an online consent for a protocol approved by the University of Georgia Institutional Review Board. After consenting, participants were automatically directed to complete a series of questionnaires administered over Qualtrics that took approximately $1 \mathrm{~h}$. Subsequently, participants were scheduled to complete an online clinical interview via Zoom, which lasted approximately 10-20 min during which the semi-structured interview for the Brief Negative Symptom Scale (BNSS) [32, 61, 61] was completed. Interviews were completed by graduate students or laboratory staff trained to reliability standards (alpha $>0.80$ ) using gold standard training videos developed by the authors of the BNSS. Participants received a $\$ 40$ check payment for participating.

Online questionnaires covered a range of content: demographics, COVID-19 health and safety behaviors, environmental factors, positive symptoms, general symptoms (e.g., anxiety, depression, and sleep), internet/social media use, and protective factors. Only the negative symptom data are the focus of this report.

Original pre-pandemic interviews took place in-person. The mean interval between pre-pandemic and during pandemic interviews was 389.86 days ( $\mathrm{SD}=223.80$ days) for CHR and 698.55 days ( $\mathrm{SD}=191.80$ days) for SZ. CN participants were not rated pre-pandemic.

All procedures were approved by the University of Georgia Institutional Review Board and the study was performed in accordance with the ethical standards laid down in the 1964 Declaration of Helsinki and its later amendments.

\section{Measures}

\section{Brief negative symptom scale (BNSS)}

The BNSS is a 13-item clinical rating scale [32]. It is rated after a 10-15 min semi-structured interview with suggested probes. The BNSS was developed following the 2005 NIMH Consensus Development Conference in response to the NIMH MATRICS initiative [33]. The 13 items cover the 5 consensus domains identified in the consensus conference.
The anhedonia items distinguish between frequency and intensity of past week pleasure, as well as intensity of future pleasure expected in relation to social, physical, recreational, and work/school domains. Avolition and asociality domains each have separate items for internal experience (i.e., wanting/desire for engaging in goal-directed activities or social interactions) and behavior (i.e., engaging in goal-directed or social activity). Blunted affect is assessed in relation to facial affect, vocal affect, and expressive body gestures. Alogia is evaluated in relation to quantity of speech and spontaneous elaboration. The BNSS also rates a 6th domain, lack of normal distress (i.e., reductions in intensity and frequency of negative emotional experience). Psychometric properties of the BNSS have previously been established in SZ, CHR, and $\mathrm{CN}$ populations, demonstrating good inter-rater agreement, internal consistency, test-retest reliability, convergent validity, and discriminant validity $[58,61,65]$. Confirmatory factor analyses and network analysis indicate that the BNSS yields both a 5-factor (anhedonia, avolition, asociality, blunted affect, alogia and hierarchical structure (i.e., two second-order high-level factors consisting of diminished expression [EXP] and motivation and pleasure [MAP], as well as 5 first-order lower level factors consisting of the 5 consensus domains: anhedonia, avolition, asociality, blunted affect, and alogia in both SZ and CHR [63], [2], [8]. As such, analyses focused on both the 5 domains and 2 dimensions.

\section{Data analysis}

Hypothesis 1 BNSS data were first analyzed for pre-pandemic differences using a 2 Group $(\mathrm{CHR}, \mathrm{SZ}) \times 5$ Domain (anhedonia, avolition, asociality, blunted affect, alogia) mixed-models ANOVA. The analysis was also repeated using the 2 dimensions (MAP, EXP) as the within-subjects variable.

Hypothesis 2 Separate 2 Time $\times 5$ Domain mixed models ANOVAs were conducted for each study to examine domain exacerbations in SZ and CHR groups throughout the pandemic. The analysis was also repeated using the 2 dimensions (MAP, EXP) as the within-subjects variable. Significant interactions were followed up within group paired samples $t$ tests comparing domains/dimensions across time intervals.

To evaluate differences in the magnitude of pandemicrelated changes between SZ and CHR groups, during prepandemic difference scores were calculated for all 5 BNSS domains and the 2 dimensions. Difference scores were entered into separate one-way ANOVAs calculated for each domain.

Hypothesis 3 Similar models were run to evaluate itemlevel hypotheses using a 2 time $\times 13$ item mixed models ANOVA. Significant interactions were followed up by post 
hoc one-way ANOVAs within each time interval, as well as within group paired samples $t$ tests comparing items across time intervals.

Hypothesis 4 Using during pandemic scores, separate 2 group $\times 5$ domain (and 2 dimension) mixed models ANOVAs were calculated for each study to determine if clinical groups displayed greater negative symptoms than CN. Significant interactions were followed up by post hoc one-way ANOVAs within each time interval.

\section{Results}

\section{Preliminary analyses}

Self-reported medication and treatment data pre- and during pandemic are reported for CHR and SZ groups in Table 2.

\section{Hypothesis 1-Pre-Pandemic}

The 2 group $\times 5$ domain mixed models ANOVA indicated a nonsignificant interaction $(F[1,54]=3.78, p=0.057)$, nonsignificant effect of group $(F[1,54]=0.58, p=0.45)$, and significant effect of domain $(F[1,54]=16.70, p<0.001)$.

Similar results emerged using the 2 broader MAP and EXP dimensions: with a nonsignificant interaction $(F[4,54]=1.25, p=0.29)$, nonsignificant effect of group $(F[1,54]=0.58, p=0.45)$, and significant effect of domain $(F[4,54]=16.71, p<0.001)$.

SZ and CHR did not differ in severity of BNSS ratings pre-pandemic. Across domains, scores tended to be lowest for alogia and highest for avolition and anhedonia. Across dimensions, scores were higher for MAP than EXP (Table 3).

\section{Hypothesis 2-pre-pandemic to pandemic changes}

Sample 1 (SZ) The 2 time $\times 5$ domain repeated measures ANOVA revealed a nonsignificant interaction $(F[4,29]=2.05, p=0.09)$, significant effect of domain $(F[4,29]=9.92, p<0.001)$, and significant effect of time $(F[1,29]=15.89, p<0.001)$. Post hoc within-group pairedsamples $t$ tests indicated that SZ had significantly higher BNSS domain scores during the pandemic compared to pre-pandemic for all domains $(t \geq 2.52, p \leq 0.017)$, except blunted affect (Similar results emerged for the 2 dimensions: nonsignificant interaction $(F[4,29]=0.01, p=0.90)$, significant effect of Dimension $(F[4,29]=4.28, p<0.05)$, and significant effect of Time $(F[1,29]=12.62, p<0.001)$. Within-group paired samples $t$ tests indicated that SZ had significantly higher MAP $(t=3.52, p<0.001)$ and EXP $(t=2.93, p<0.01)$ scores during the pandemic compared to pre-pandemic. Thus, negative symptoms worsened during the pandemic in SZ.

$$
t=1.72, p=0.096) \text {. }
$$

Sample $2($ CHR $)$ The 2 Time $\times 5$ Domain mixedmodels ANOVA revealed a nonsignificant interaction $(F[4,22]=0.17, p=0.95)$, significant effect of Domain $(F[4,22]=14.36, p<0.001)$, and nonsignificant effect of Time $(F[1,22]=1.70, p=0.21)$.

Similarly, the dimensional analyses indicated a nonsignificant interaction $(F[1,22]=0.25, p=0.63)$, significant effect of Dimension $(F[1,22]=23.50, p<0.001)$, and nonsignificant effect of Time $(F[1,22]=0.47, p<0.50)$. Thus, negative symptoms did not globally worsen during the pandemic in CHR.
Table 2 Medication and Treatment Data Pre and DuringPandemic

\begin{tabular}{llll}
\hline & SZ & CHR & $X^{2}, p$ value \\
\hline \% Taking medications for any psychiatric conditions & & & \\
Pre-pandemic & $93 \%$ & $52 \%$ & $13.2, p<0.001$ \\
During pandemic & $90 \%$ & $60 \%$ & $6.8, p=0.01$ \\
\% Reporting they missed taking medications for any reason & & & \\
Pre-pandemic & $21 \%$ & $8 \%$ & $2.03, p=0.27$ \\
During pandemic & $24 \%$ & $24 \%$ & $0.00, p=1.0$ \\
\% Missing scheduled appointments with healthcare providers & & & \\
Pre-pandemic & $17 \%$ & $8 \%$ & $1.3, p=0.44$ \\
$\quad$ During PANDEMIC & $37 \%$ & $28 \%$ & $.47, p=0.57$ \\
\% With access to remote healthcare (e.g., teletherapy/video therapy) & & & \\
$\quad$ Pre-pandemic & $23 \%$ & $4 \%$ & $5.65, p=0.03$ \\
During pandemic & $83 \%$ & $44 \%$ & $9.3, p=0.004$ \\
\hline
\end{tabular}

$S Z$ schizophrenia, $C H R$ clinical high-risk for psychosis 
Table 3 Brief Negative Symptom Scale (BNSS) scores in clinical and control groups pre- and during-pandemic

\begin{tabular}{|c|c|c|c|c|c|c|}
\hline & \multicolumn{6}{|c|}{ Study 1 Study 2} \\
\hline & \multicolumn{2}{|l|}{$\mathrm{SZ}$} & \multirow{2}{*}{$\begin{array}{l}\mathrm{CN} \\
\text { During }\end{array}$} & \multicolumn{2}{|l|}{ CHR } & \multirow{2}{*}{$\begin{array}{l}\mathrm{CN} \\
\text { During }\end{array}$} \\
\hline & Pre & During & & Pre & During & \\
\hline MAP & $1.23(1.17)$ & $2.11(1.54)$ & $0.86(1.01)$ & $1.28(0.54)$ & $1.44(1.05)$ & $0.75(0.86)$ \\
\hline EXP & $0.82(1.08)$ & $1.57(1.41)$ & $0.64(1.44)$ & $0.47(0.79)$ & $0.6(0.91)$ & $0.47(0.72)$ \\
\hline Anhedonia & $1.23(1.51)$ & $2.12(1.72)$ & $0.91(1.16)$ & $1.43(0.9)$ & $1.48(1.29)$ & $0.74(0.91)$ \\
\hline Asociality & $1.02(1.29)$ & $1.72(1.56)$ & $0.87(1.05)$ & $1.02(0.9)$ & $1.17(1.04)$ & $0.72(0.79)$ \\
\hline Avolition & $1.45(1.28)$ & $2.5(1.8)$ & $0.78(1.18)$ & $1.31(0.97)$ & $1.67(1.26)$ & $0.79(1.35)$ \\
\hline Blunted affect & $1.19(1.44)$ & $1.67(1.31)$ & $0.49(1.01)$ & $0.65(1.08)$ & $0.71(1.12)$ & $0.47(0.75)$ \\
\hline Alogia & $0.27(0.81)$ & 1.47 (1.68) & $0.58(1.46)$ & $0.19(0.43)$ & $0.45(0.86)$ & $0.5(0.81)$ \\
\hline
\end{tabular}

All values are mean with SD in parentheses

$B N S S$ Brief Negative Symptom Scale, $C H R$ clinical high-risk, $C N$ control, SZ schizophrenia, MAP motivation and pleasure, EXP diminished expression, Pre pre-pandemic, During during pandemic, Note $\mathrm{CN}$ were only evaluated during the pandemic in both studies

\section{Hypothesis 3-item-level effects}

Item-level analyses were conducted to evaluate hypothesized differences in effects for experiential versus behavioral components of negative symptoms.

Sample $1(\mathbf{S Z})$ The 2 Time $\times 12$ Item-level mixed models ANOVA indicated a nonsignificant interaction $(F[11,22]=1.23, p<0.27)$, significant main effect of Item $(F[11,21]=4.07, p<0.001)$, and significant main effect of Time $(F[1,22]=13.08, p<0.01)$. Post hoc within group paired samples $t$ tests indicated significantly higher scores during the pandemic compared to pre for items: one (intensity of pleasure during activities), two (Frequency of pleasurable activities), three (intensity of expected pleasure from future activities), five (asociality behavior), seven (avolition behavior), eight (avolition internal experience), ten (vocal expression), twelve (quantity of speech), and thirteen (spontaneous elaboration) ( $p<0.05$ for all). Effects were nonsignificant for items: six (asociality internal experience, $p=0.058)$, nine ( $F$ acial expression, $p=0.29)$, and eleven (expressive gestures, $p=0.065$ ).

Sample $2(\mathbf{C H R}) \quad$ The 2 Time $\times 12$ Item-level mixed models ANOVA indicated a significant interaction $(F[11,20]=3.06, p<0.001)$, significant main effect of Item $(F[11,20]=9.65, p<0.001)$, and nonsignificant main effect of Time $(F[1,20]=1.50, p=0.24)$. Post hoc within group paired samples $t$ tests indicated significantly higher scores during the pandemic compared to pre for items: one (intensity of pleasure during activities, $p=0.013$ ), two (Frequency of pleasurable activities, $p=0.021$ ), three (intensity of expected pleasure from future activities, $p=0.029$ ), and five (asociality behavior, $p=0.02$ ). Effects were nonsignificant for items: six (asociality internal experience, $p=0.85$ ), seven (avolition behavior, $p=0.11$ ), eight (avolition internal experience, $p=1.00$ ), nine ( $F$ acial expression, $p=0.51$ ), ten (vocal expression, $p=0.78$ ), eleven (expressive gestures, $p=0.40$ ), twelve (quantity of speech, $p=0.26$ ), and thirteen (spontaneous elaboration, $p=0.14$ ). Thus, CHR experienced increases in all 3 anhedonia items and the asociality behavior item during the pandemic.

\section{Hypothesis 4-pandemic group differences}

Sample 1 (SZ) The 2 Group $\times 5$ BNSS domain mixed models ANOVA revealed a nonsignificant interaction $(F[4,57]=2.07, p=0.085)$, significant main effect of Group $(F[1,57]=16.80, p<0.001)$, and main effect of Domain $(F[4,57]=7.08, p<0.001)$. Post hoc one-way ANOVAs indicated that $\mathrm{SZ}$ had significantly higher ratings than $\mathrm{CN}$ for all 5 domains: anhedonia $(F=10.17, p=0.002)$, asociality $(F=6.11, p=0.016)$, avolition $(F=19.15, p<0.001)$, blunted affect $(F=14.78, p<0.001)$, and alogia $(F=4.73$, $p=0.034)$.

The 2 Group $\times 2$ Dimension mixed models ANOVA indicated a nonsignificant interaction $(F[1,58]=0.45, p=0.51)$, significant main effect of $\operatorname{Group}(F[1,58]=10.70, p=0.002)$, and main effect of Dimension $(F[1,58]=2.78, p=0.10)$. One way ANOVA indicated that SZ had significantly higher scores than CN on MAP $(F[1,58]=13.29, p<0.001)$ and $\operatorname{EXP}(F[1,58]=6.37, p=0.014)$ during the pandemic.

Sample 2 (CHR) The 2 Group $\times 5$ BNSS domain mixed models ANOVA revealed a significant interaction $(F[4,52]=3.25, p<0.013)$, main effect of Group $(F[1,52]=4.70, p=0.035)$, and main effect of Domain $(F[4,52]=9.88, p<0.001)$. Post hoc one-way ANOVAs indicated that $\mathrm{CHR}$ had significantly higher ratings than $\mathrm{CN}$ for anhedonia $(F=6.85, p=0.012)$ and avolition $(F=5.63$, $p=0.021)$, but not asociality $(F=2.96, p=0.091)$, blunted affect $(F=1.0, p=0.32)$, or alogia $(F=0.01, p=0.91)$.

The 2 Group $\times 2$ Dimension mixed models ANOVA indicated a significant interaction $(F[1,52]=4.61, p=0.037)$, 
trend toward a main effect of Group $(F[1,52]=3.45$, $p=0.069)$, and significant main effect of Dimension $(F[1,52]=18.12, p<0.001)$. One way ANOVA indicated that $\mathrm{SZ}$ had significantly higher scores than $\mathrm{CN}$ on MAP $(F[1,52]=6.55, p<0.013)$, but groups did not differ on EXP $(F[1,52]=0.40, p=0.53)$.

\section{Discussion}

Results supported hypotheses in the SZ group, which exhibited global exacerbations in negative symptoms during the COVID-19 pandemic. Symptom worsening was true of both the EXP and MAP dimensions. A more fine-grained analysis of the 5 domains indicated that increases were present in $4 / 5$ domains, including anhedonia, avolition, asociality, and alogia. Increases in blunted affect were more marginal and at a trend level. Item-level analyses confirmed that symptom exacerbations were not simply tautological and a reflection of behavioral restrictions imposed by COVID safety precautions.

A different pattern emerged in the CHR group which did not evidence global increases in the 2 dimensions or 5 domains. Item-level analyses indicated that CHR did indeed exhibit increased severity on all 3 anhedonia items and the asociality behavior item; however, differences across time were nonsignificant for other items. Notably, CHR evidenced greater global negative symptom severity than $\mathrm{CN}$ during the pandemic; however, not all dimensions and domains were significantly higher in CHR. The MAP dimension was significantly higher in CHR than $\mathrm{CN}$, but groups did not differ on EXP. Domain level analyses clarified that CHR were significantly higher than $\mathrm{CN}$ on anhedonia and avolition, but not asociality, blunted affect, or alogia. To our knowledge, comparisons between CHR and demographically matched $\mathrm{CN}$ have not been reported in prior studies. However, this pattern of results differs from the SZ sample, which was rated higher than $\mathrm{CN}$ for both dimensions and all 5 domains during the pandemic.

The different patterns of results observed between the two clinical samples raises an important question: why did SZ display global increases in negative symptoms during the COVID-19 pandemic but CHR did not? The two groups did not differ in negative symptom severity pre-pandemic making a greater pre-existing degree of psychopathology in SZ an unlikely explanation. Differences in medication and treatment regimen also seem an unlikely explanation. If these factors were accounting for the effect, one would expect the CHR group to receive more medication and treatment than SZ. However, the opposite was true. In addition, the CHR group was more likely to stop taking medications during the pandemic, whereas this did not occur as frequently in SZ.
Several other explanations may be plausible. First, although the magnitude of severity does not differ between SZ and CHR pre-pandemic, chronicity undoubtedly does. Although we do not have longitudinal data from illness onset to confirm this speculation, it is reasonable to assume that our SZ sample would have manifested negative symptoms for 1-2 decades on average based on the mean group age, whereas these symptoms would likely have emerged much more recently in CHR. Greater chronicity may lead negative symptoms to be more entrenched in SZ and, therefore, more likely to increase following extreme behavioral reduction, such as what is occurring during the pandemic.

Second, age and greater chronicity likely contribute to very different environmental influences on negative symptoms in SZ and CHR. There is evidence that certain environmental deprivation factors are associated with negative symptoms in SZ, including under-stimulating environments, smaller social networks, aberrant family social dynamics (e.g., cohesion, positive emotion expression), greater local income inequality, lower socio-economic status, and receiving minimal care and attention in group homes $[73,7,28$, $47,48,53,69,70]$. It is possible that these environmental factors are less impoverished in CHR and, therefore, less impactful on negative symptoms during the pandemic. Perhaps CHR demonstrate less robust negative symptom exacerbations during the pandemic, because their environmental microsystems are more intact (i.e., greater connectedness with family, friends, school, work) or they are better able to navigate electronic communications to maintain social ties (e.g., social media, text, video calls), leading to greater buffering against symptom exacerbation.

Third, another explanation is that the factors driving negative symptoms differ substantially between CHR and SZ samples, even if pre-pandemic scores do not differ. It is possible for two participants to receive the same score on a negative symptom rating scale, such as the BNSS, for very different reasons. For some participants, higher scores can reflect primary manifestations of the illness (i.e., negative symptoms are idiopathic and assumed to be driven by mechanisms inherent to apathy). However, for other participants, the same score may be achieved due to secondary sources of negative symptoms, such as depression, anxiety, hallucinations, or delusions. It is unclear whether SZ and CHR populations differ in the relative proportion of negative symptom cases that are due to primary or secondary sources. However, it stands to reason that CHR may be more likely to have a greater preponderance of secondary negative symptoms than SZ due to the higher rates of depression and anxiety [1]. Perhaps the COVID-19 pandemic is having a greater impact on primary negative symptom profiles that tend to be persistent, trait-like features of the illness.

Fourth, it is unclear whether psychological and neurobiological mechanisms underlying negative symptoms differ 
between SZ and CHR populations. Psychological (e.g., defeatist performance beliefs, low pleasure beliefs) and neurobiological (e.g., cortico-striatal dysfunction, reward processing abnormalities, inflammation) mechanisms underlying negative symptoms are becoming increasingly wellunderstood in SZ [20, 22, 26, 59]. However, it is unclear whether these mechanisms also contribute to negative symptoms in CHR. Preliminary evidence has implicated defeatist performance beliefs [11], reward processing impairments $[3,4,43,63]$, and inflammatory abnormalities [16] in CHR, however, it is unclear whether those abnormalities are more pronounced in CHR converters than non-converters or those with persistent negative symptom profiles. Preliminary evidence does suggest some important differences between SZ and CHR, such as the presence of true hedonic deficits in CHR that are not present in SZ [63]. It is possible that different mechanisms are contributing to negative symptoms in CHR than SZ that are driven by differences in the proportion of secondary negative symptoms.

Certain limitations should be considered. First, BNSS ratings were conducted in person pre-pandemic and over video calls during the pandemic. It is unclear whether or how the difference in rating platform may have influenced results. We suspect that the experience of completing an interview remotely over the internet may be more cognitively taxing than in-person interviews. Online, individuals seem more likely to monitor their own expressions in their video window and experience fatigue more rapidly, perhaps due to greater demands on cognitive control and shifting attention between their own video window and the interviewer's. It has been demonstrated that taxing working memory resources can exacerbate blunted facial affect, vocal affect, and alogia in SZ. Although blunted affect was the domain that increased the least during the pandemic in our SZ sample, the impact of the video platform exacerbating expressive negative symptoms during pandemic ratings cannot be dismissed. Second, conducting a remote study during the pandemic proved challenging in several ways. Not all participants who were invited to participate elected to do so and the sample tended to have more females and higher functioning participants than what would be typical for a study conducted in-person in our lab. Findings may, therefore, not generalize to all individuals with the conditions studied. Third, due to the online nature of the study, we were unable to explore potential neurobiological mechanisms contributing to negative symptom changes during the pandemic. Fourth, healthy $\mathrm{CN}$ did not receive BNSS ratings pre-pandemic. Although not interviewing $\mathrm{CN}$ is a common practice in the field, because they rarely exhibit negative symptoms, this prevented comparisons to pre-pandemic status. Finally, ratings were taken at but one point, approximately 3-6 months after the COVID-19 pandemic began. It is unclear whether the negative symptom exacerbations we observed here would have been greater or lesser than those at other timepoints during the pandemic. However, we do plan to follow-up these participants longitudinally to see if symptom exacerbations have remitted after the COVID-19 pandemic has subsided.

Despite these limitations, several conclusions can be drawn. Negative symptoms are increasing during the pandemic in SZ, with increases observed globally across dimensions and domains. Negative symptom exacerbations have been less pronounced in CHR, who demonstrated primary increases in the domains of anhedonia and avolition. Factors underlying these pandemic-related symptom exacerbations warrant further study. The COVID19 pandemic provides a unique opportunity to observe the effects of widespread environmental deprivation on symptoms. Although restrictions to social microsystem environments would be largely unheard of for the general population during normal times, the sudden environmental change has elements in common with the emergence of psychosis, providing insight into how impoverished environments lead to exacerbations in negative symptoms throughout the global population. These effects appear profound and highlight the need to assess environmental contributions to negative symptoms during normal times (Strauss, in press). If present, they could be targeted using systemslevel therapies, such as multi-systemic treatment [29]. Finally, given how long the pandemic has persisted, many research labs, pharmaceutical companies, and clinicians are forced with deciding how to assess symptoms using alternative means. These findings suggest that negative symptom ratings can be validly conducted remotely using video-based interviews during the pandemic.

Funding Research was supported by NIMH Grants R21-MH112925, R21-MH122863, R21-MH122863, and R01MH116039 to Dr. Strauss.

\section{Declarations}

Conflict of interest G. P. Strauss is one of the original developers of the Brief Negative Symptom Scale (BNSS) and receives royalties and consultation fees from ProPhase LLC in connection with commercial use of the BNSS and other professional activities; these fees are donated to the Brain and Behavior Research Foundation. Dr. Strauss has received honoraria and travel support from ProPhase LLC for training pharmaceutical company raters on the BNSS. In the past two years, Dr. Strauss has consulted for and/or been on the speaker bureau for 
Minerva Neurosciences, Acadia, and Lundbeck pharmaceutical companies. All other authors have no conflicts to report.

\section{References}

1. Addington J, Piskulic D, Liu L, Lockwood J, Cadenhead KS, Cannon TD, Tsuang MT (2017) Comorbid diagnoses for youth at clinical high risk of psychosis. Schizophr Res 190:90-95

2. Ahmed AO, Kirkpatrick B, Galderisi S, Mucci A, Rossi A, Bertolino A, Hartmann-Riemer MN (2019) Cross-cultural validation of the 5-factor structure of negative symptoms in schizophrenia. Schizophr Bull 45(2):305-314

3. Bartolomeo LA, Chapman HC, Raugh IM, Strauss GP (2020) Delay discounting in youth at clinical high-risk for psychosis and adults with schizophrenia. Psychol Med: 1-8.

4. Bartolomeo LA, Erickson MA, Arnold LE, Strauss GP (2019) Frontal alpha asymmetry in youth at clinical high risk for psychosis. Curr Behav Neurosci Rep 6(2):21-26

5. Bo HX, Li W, Yang Y, Wang Y, Zhang Q, Cheung T, Xiang YT (2020) Posttraumatic stress symptoms and attitude toward crisis mental health services among clinically stable patients with COVID-19 in China. Psychol Med:1-2.

6. Brown E, Gray R, Monaco SL, O’Donoghue B, Nelson B, Thompson A, McGorry P (2020) The potential impact of COVID-19 on psychosis: a rapid review of contemporary epidemic and pandemic research. Schizophr Res 222:79-87

7. Canive JM, Sanz-Fuentenebro J, Vazquez C, Qualls C, Fuentenebro F, Tuason VB (1995) Family environment predictors of outcome in schizophrenic patients in Spain: a nine-month follow-up study. Acta Psychiatr Scand 92(5):371-377

8. Chang WC, Strauss GP, Ahmed AO, Wong SC, Chan JK, Lee EH, Chen EY (2020) The latent structure of negative symptoms in individuals with attenuated psychosis syndrome and early psychosis: Support for the 5 consensus domains. Schizoph Bull.

9. Charlson FJ, Ferrari AJ, Santomauro DF, Diminic S, Stockings E, Scott JG, Whiteford HA (2018) Global epidemiology and burden of schizophrenia: findings from the global burden of disease study 2016. Schizophr Bull 44(6):1195-1203

10. Choi KH, Jaekal E, Lee GY (2016) Motivational and behavioral activation as an adjunct to psychiatric rehabilitation for mild to moderate negative symptoms in individuals with schizophrenia: a proof-of-concept pilot study. Front Psychol 7:1759

11. Clay KB, Raugh IM, Bartolomeo LA, Strauss GP (2020) Defeatist performance beliefs in individuals at clinical high-risk for psychosis and outpatients with chronic schizophrenia. Early Intervent Psychiatry.

12. Cohen AS, Cowan T, Le TP, Schwartz EK, Kirkpatrick B, Raugh IM, Strauss GP (2020) Ambulatory digital phenotyping of blunted affect and alogia using objective facial and vocal analysis: proof of concept. Schizophr Res.

13. Czeisler MÉ, Lane RI, Petrosky E, Wiley JF, Christensen A, Njai R, Czeisler CA (2020) Mental health, substance use, and suicidal ideation during the COVID-19 pandemic-United States, June 24-30, 2020. Morb Mortal Wkly Rep 69(32):1049

14. D'Agostino A, D'Angelo S, Giordano B, Cigognini AC, Chirico ML, Redaelli C, Gambini O (2020) Brief Psychotic Disorder during the national lockdown in Italy: an emerging clinical phenomenon of the coronavirus pandemic. Schizophr Bull. https:// doi.org/10.1093/schbul/sbaa112

15. Dean DJ, Tso IF, Giersch A, Lee HS, Baxter T, Griffith T, Park S (2020) Cross-Cultural comparisons of psychosocial distress in the USA, South Korea, France, and Hong Kong during the initial phase of COVID-19. Psychiatry Res. https://doi.org/10. 1016/j.psychres.2020.113593

16. Delaney S, Fallon B, Alaedini A, Yolken R, Indart A, Feng T, Javitt D (2019) Inflammatory biomarkers in psychosis and clinical high risk populations. Schizophr Res 206:440-443

17. DeLuca JS, Andorko ND, Chibani D, Jay SY, Rakhshan Rouhakhtar PJ, Petti E, Akouri-Shan L (2020) Telepsychotherapy with youth at clinical high risk for psychosis: Clinical issues and best practices during the COVID-19 pandemic. J Psychother Integr 30(2):304. https://doi.org/10.1037/int0000211

18. Dirlikov E, Fechter-Leggett E, Thorne SL, Worrell CM, SmithGrant JC, Chang J, Aden T (2020) CDC Deployments to state, tribal, local, and territorial health departments for COVID-19 emergency public health response-United States, January 21July 25, 2020. Morb Mortal Wkly Rep 69(39):1398

19. Fervaha G, Foussias G, Agid O, Remington G (2014) Motivational and neurocognitive deficits are central to the prediction of longitudinal functional outcome in schizophrenia. Acta Psychiatr Scand 130(4):290-299

20. First MB (2014) Structured clinical interview for the DSM (SCID). Encyclop Clin Psychol:1-6.

21. First MB, Williams JB, Benjamin LS, Spitzer RL (2016) SCID5-PD: structured clinical interview for DSM-5® personality disorders. American Psychiatric Association Publishing.

22. Goldsmith DR, Haroon E, Miller AH, Strauss GP, Buckley PF, Miller BJ (2018) TNF- $\alpha$ and IL- 6 are associated with the deficit syndrome and negative symptoms in patients with chronic schizophrenia. Schizophr Res 199:281-284

23. Granholm E, Holden J, Worley M (2018) Improvement in negative symptoms and functioning in cognitive-behavioral social skills training for schizophrenia: mediation by defeatist performance attitudes and asocial beliefs. Schizophr Bull 44(3):653-661

24. Granholm E, Holden J, Dwyer K, Mikhael T, Link P, Depp C (2020) Mobile-assisted cognitive behavioral therapy for negative symptoms: open single-arm trial with schizophrenia patients. JMIR Mental Health 7(12):e24406

25. Granholm E, Holden J, Link PC, McQuaid JR (2014) Randomized clinical trial of cognitive behavioral social skills training for schizophrenia: improvement in functioning and experiential negative symptoms. J Consult Clin Psychol 82(6):1173

26. Grant PM, Beck AT (2009) Defeatist beliefs as a mediator of cognitive impairment, negative symptoms, and functioning in schizophrenia. Schizophr Bull 35(4):798-806

27. Gruber J, Prinstein MJ, Clark LA, Rottenberg J, Abramowitz JS, Albano AM, Forbes EE (2020) Mental health and clinical psychological science in the time of COVID-19: challenges, opportunities, and a call to action. Am Psychol. https://doi.org/ 10.1037/amp0000707

28. Halford WK (1991) Beyond expressed emotion: behavioral assessment of family interaction associated with the course of schizophrenia. Behav Assess

29. Henggeler SW, Schoenwald SK, Borduin CM, Rowland MD, Cunningham PB (2009) Multisystemic therapy for antisocial behavior in children and adolescents. Guilford Press

30. Heymann DL, Shindo N (2020) COVID-19: what is next for public health? The Lancet 395(10224):542-545

31. Holmes EA, O'Connor RC, Perry VH, Tracey I, Wessely S, Arseneault L, Ford T (2020) Multidisciplinary research priorities for the COVID-19 pandemic: a call for action for mental health science. Lancet Psychiatry. https://doi.org/10.1016/ S2215-0366(20)30168-1

32. Kirkpatrick B, Strauss GP, Nguyen L, Fischer BA, Daniel DG, Cienfuegos A, Marder SR (2011) The brief negative symptom scale: psychometric properties. Schizophr Bull 37(2):300-305 
33. Kirkpatrick B, Fenton WS, Carpenter WT, Marder SR (2006) The NIMH-MATRICS consensus statement on negative symptoms. Schizophr Bull 32(2):214-219

34. Klingberg S, Wölwer W, Engel C, Wittorf A, Herrlich J, Meisner C, Wiedemann G (2011) Negative symptoms of schizophrenia as primary target of cognitive behavioral therapy: results of the randomized clinical TONES study. Schizophr Bull 37(suppl_2): S98-S110

35. Kozloff N, Mulsant BH, Stergiopoulos V, Voineskos AN (2020) The COVID-19 global pandemic: implications for people with schizophrenia and related disorders. Schizophr Bull. https://doi. org/10.1093/schbul/sbaa051

36. Krendl AC, Perry BL (2020) The impact of sheltering in place during the COVID-19 pandemic on older adults' social and mental well-being. J Gerontol Ser B Psychol Sci Soc Sci

37. Lai J, Ma S, Wang Y, Cai Z, Hu J, Wei N, Tan H (2020) Factors associated with mental health outcomes among health care workers exposed to coronavirus disease 2019. JAMA Netw Open 3(3):e203976-e203976. https://doi.org/10.1001/jaman etworkopen.2020.3976

38. Lasry A, Kidder D, Hast M, Poovey J, Sunshine G, Zviedrite N, Ethier KA (2020) Timing of cmmunity mitigation and changes in reported COVID-19 and community mobility-four US metropolitan areas, February 26-April 1, 2020.

39. Lee HS, Dean D, Baxter T, Griffith T, Park S (2020) Deterioration of mental health despite successful control of the COVID19 pandemic in South Korea. Psychiatry Res:113570.

40. Lynch DA, Medalia A, Saperstein A (2020) The design, implementation, and acceptability of a telehealth comprehensive recovery service for people with complex psychosis living in NYC during the COVID-19 crisis. Front Psych 11:926. https:// doi.org/10.3389/fpsyt.2020.581149

41. Mazza MG, De Lorenzo R, Conte C, Poletti S, Vai B, Bollettini I, Benedetti F (2020) Anxiety and depression in COVID-19 survivors: role of inflammatory and clinical predictors. Brain Behav Immun 89:594-600. https://doi.org/10.1016/j.bbi.2020. 07.037

42. Miller TJ, McGlashan TH, Woods SW, Stein K, Driesen N, Corcoran CM, Davidson L (1999) Symptom assessment in schizophrenic prodromal states. Psychiatr Q 70(4):273-287

43. Millman ZB, Gallagher K, Demro C, Schiffman J, Reeves GM, Gold JM, Buchanan RW (2019) Evidence of reward system dysfunction in youth at clinical high-risk for psychosis from two event-related fMRI paradigms. Schizophr Res.

44. Miu AS, Vo HT, Palka JM, Glowacki CR, Robinson RJ (2020) Teletherapy with serious mental illness populations during COVID-19: telehealth conversion and engagement. Couns Psychol Q. https://doi.org/10.1080/09515070.2020.1791800

45. Nguyen AX, Gervasio KA, Wu AY (2020) COVID-19 recommendations from ophthalmic and plastic reconstructive surgery societies worldwide. Ophthalmic Plast Reconstr Surg 36(4):334

46. O'Connor DB, Aggleton JP, Chakrabarti B, Cooper CL, Creswell C, Dunsmuir S, Jones MV (2020) Research priorities for the COVID-19 pandemic and beyond: a call to action for psychological science. Br J Psychol 111(4):603-629. https://doi.org/10.1111/ bjop. 12468

47. Oshima I, Mino Y, Inomata Y (2005) Effects of environmental deprivation on negative symptoms of schizophrenia: a nationwide survey in Japan's psychiatric hospitals. Psychiatry Res 136(2-3):163-171

48. Ove RH, Lisbeth RK, Gustaf RNK (2004) Living in a group dwelling: how do residents spend their time in a psychiatric group dwelling? Int J Nurs Stud 41(6):651-659

49. Piskulic D, Addington J, Cadenhead KS, Cannon TD, Cornblatt BA, Heinssen R, Woods SW (2012) Negative symptoms in individuals at clinical high risk of psychosis. Psychiatry Res 196(2-3):220-224

50. Quinn A, Laws M (2020) Addressing community needs and preparing for the secondary impacts of Covid-19. Nejm Catal Innov Care Deliv.

51. Raugh IM, James SH, Gonzalez CM, Chapman HC, Cohen AS, Kirkpatrick B, Strauss GP (2020) Geolocation as a digital phenotyping measure of negative symptoms and functional outcome. Schizophr Bull 46(6):1596-1607

52. Rector NA, Beck AT (2001) Cognitive behavioral therapy for schizophrenia: an empirical review. J Nerv Ment Dis 189(5):278-287

53. Samele C, van Os J, McKenzie K, Wright A, Gilvarry C, Manley C, UK700 Group (2001) Does socioeconomic status predict course and outcome in patients with psychosis? Soc Psychiatry Psychiatr Epidemiol 36(12):573-581

54. Schuchat A (2020) Public health response to the initiation and spread of pandemic COVID-19 in the United States, February 24-April 21, 2020. In: MMWR. Morbidity and mortality weekly report, 69.

55. Shaukat N, Ali DM, Razzak J (2020) Physical and mental health impacts of COVID-19 on healthcare workers: a scoping review. Int J Emerg Med 13(1):1-8. https://doi.org/10.1186/ s12245-020-00299-5

56. Sher L (2020) The impact of the COVID-19 pandemic on suicide rates. QJM: Int J Med 113(10): 707-712. https://doi.org/10.1093/ qjmed/hcaa202

57. Strauss GP (2021) A bioecosystem theory of negative symptoms in schizophrenia. Front Psychiatry (in press)

58. Strauss GP, Chapman HC (2018) Preliminary psychometric properties of the brief negative symptom scale in youth at clinical high-risk for psychosis. Schizophr Res 193:435-437

59. Strauss GP, Cohen AS (2017) A transdiagnostic review of negative symptom phenomenology and etiology. Schizophr Bull 43(4):712-719

60. Strauss GP, Harrow M, Grossman LS, Rosen C (2010) Periods of recovery in deficit syndrome schizophrenia: A 20-year multifollow-up longitudinal study. Schizophr Bull 36(4):788-799

61. Strauss GP, Hong LE, Gold JM, Buchanan RW, McMahon RP, Keller WR, Kirkpatrick B (2012) Factor structure of the brief negative symptom scale. Schizophr Res 142(1-3):96-98

62. Strauss GP, Keller WR, Buchanan RW, Gold JM, Fischer BA, McMahon RP, Kirkpatrick B (2012) Next-generation negative symptom assessment for clinical trials: validation of the Brief Negative Symptom Scale. Schizophr Res 142(1-3):88-92

63. Strauss GP, Nuñez A, Ahmed AO, Barchard KA, Granholm E, Kirkpatrick B, Allen DN (2018) The latent structure of negative symptoms in schizophrenia. JAMA Psychiat 75(12):1271-1279

64. Strauss GP, Sandt AR, Catalano LT, Allen DN (2012) Negative symptoms and depression predict lower psychological well-being in individuals with schizophrenia. Compr Psychiatry 53(8):1137-1144

65. Strauss GP, Vertinski M, Vogel SJ, Ringdahl EN, Allen DN (2016) Negative symptoms in bipolar disorder and schizophrenia: a psychometric evaluation of the brief negative symptom scale across diagnostic categories. Schizophr Res 170(2-3):285-289

66. Strauss GP, Waltz JA, Gold JM (2014) A review of reward processing and motivational impairment in schizophrenia. Schizophr Bull 40(Suppl_2): S107-S116.

67. Taquet M, Luciano S, Geddes JR, Harrison PJ (2020) Bidirectional associations between COVID-19 and psychiatric disorder: retrospective cohort studies of 62354 COVID-19 cases in the USA. Lancet Psychiatry.

68. Telford CT, Onwubiko U, Holland DP, Turner K, Prieto J, Smith S, Williams S (2020) Preventing COVID-19 outbreaks in longterm care facilities through preemptive testing of residents and 
staff members-fulton county, Georgia, March-May 2020. Morb Mortal Wkly Rep 69(37):1296

69. Thorup A, Petersen L, Jeppesen P, Øhlenschlæger J, Christensen T, Krarup G, Nordentoft M (2006) Social network among young adults with first-episode schizophrenia spectrum disorders. Soc Psychiatry Psychiatr Epidemiol 41(10):761-770

70. Tibber MS, Kirkbride JB, Mutsatsa S, Harrison I, Barnes TR, Joyce EM, Huddy V (2019) Are socioenvironmental factors associated with psychotic symptoms in people with first-episode psychosis? A cross-sectional study of a West London clinical sample. BMJ Open 9(9):e030448
71. Tso IF, Park S (2020) Alarming levels of psychiatric symptoms and the role of loneliness during the COVID-19 epidemic: a case study of Hong Kong. Psychiatry Res 293:113423

72. Vizheh M, Qorbani M, Arzaghi SM, Muhidin S, Javanmard Z, Esmaeili M (2020) The mental health of healthcare workers in the COVID-19 pandemic: a systematic review. J Diab Metab Disord: 1-12. Advance online publication. https://doi.org/10.1007/ s40200-020-00643-9

73. Wing JK, Brown GW (1970) Institutionalism and schizophrenia: a comparative study of three mental hospitals: 1960-1968. 